\title{
Target absent trials in configural contextual cuing
}

\author{
Melina A. Kunar • Jeremy M. Wolfe
}

Published online: 21 June 2011

(C) Psychonomic Society, Inc. 2011

\begin{abstract}
In contextual cuing (CC), reaction times for finding targets are faster in repeated displays than in displays that have never been seen before. This has been demonstrated using target-distractor configurations, global background colors, naturalistic scenes, and covariation of targets with distractors. The majority of CC studies have used displays in which the target is always present. This study investigated what happens when the target is sometimes absent. Experiment 1 showed that, although configural CC occurs in displays when the target is always present, there is no $\mathrm{CC}$ when the target is always absent. Experiment 2 showed that there is no CC when the same spatial layout can be both target present and target absent on different trials. The presence of distractors in locations that had contained targets on other trials appeared to interfere with $\mathrm{CC}$, and even disrupted the expression of $\mathrm{CC}$ in previously learned contexts (Exps. 3-5). These results show that targetdistractor associations are the important element in producing $\mathrm{CC}$ and that, consistent with a response selection account, changing the response type from an orientation task to a detection task removes the $\mathrm{CC}$ effect.
\end{abstract}

Keywords Attention - Visual search .

Attention and memory

M. A. Kunar $(\bowtie)$

Department of Psychology, University of Warwick,

Coventry CV4 7AL, UK

e-mail: m.a.kunar@warwick.ac.uk

J. M. Wolfe

Harvard Medical School,

Boston, MA, USA

J. M. Wolfe

Brigham and Women's Hospital,

Boston, MA, USA
Going about our daily routine, we often have to visually search the world to achieve our goals. Any given scene contains an abundance of visual information that needs to be attended, filtered, and analyzed in order to help us find what we are looking for. Take the example of searching for a friend in a crowd. If you were to attempt to pay attention to all of the people in the crowd at once, your visual system would be overwhelmed. In order to search effectively, the visual system has developed several attentional mechanisms that enable us to limit our processing to one object or, perhaps, to a small set of objects at any one time.

In the laboratory, the real-world task is typically simplified by having participants search for a target among a variable number of distractor items and recording the reaction time (RT) taken to find that target. A number of factors have been found to influence RTs during these search tasks. For example, if a target is defined by an abrupt onset (Yantis \& Jonides, 1984) or possesses a uniquely salient feature (Theeuwes, 1992; Treisman \& Gelade, 1980), attention is deployed to it rapidly. Likewise, top-down attentional sets can guide attention to stimuli that possess target features (e.g., Duncan \& Humphreys, 1989; Egeth, Virzi, \& Garbart, 1984; Wolfe, Cave, \& Franzel, 1989). More pertinent to the research in this article, the presence of a meaningful global context or scene can also affect search performance (e.g., Biederman, 1972; Reber, 1989).

Chun and Jiang (1998) showed that a familiar spatial context can speed search even if the context is meaningless, and even if the observer does not know explicitly that the context is familiar (see Chun, 2000, for a review). In their experiments, Chun and Jiang (1998) had participants search for a rotated $\mathrm{T}$ among rotated letter Ls. Unbeknownst to the participants, some of the displays were repeated over time, maintaining the same exact target-distractor configuration. 
The results showed that RTs were faster in repeated displays, where the unique target location was correlated with display configuration, than in nonrepeated displays, where the configuration was unrelated to the location of the target. The authors concluded that the repeated spatial context of the displays cued the location of the target, leading to faster search. Further studies have shown that this knowledge was implicit. Participants have often failed to explicitly recognize repeated displays (Chun \& Jiang, 1998) or to correctly identify where in the display the target has appeared (Chun \& Jiang, 2003; although see Smyth \& Shanks, 2008, who suggested that participants may have some explicit awareness). It has also been shown that the "contextual cuing" (CC) benefit develops rapidly and decays slowly. Participants showed faster RTs after only five repetitions of a display (Chun \& Jiang, 1998) and still showed a CC effect after a week (Chun \& Jiang, 2003).

There have been several theories proposed to explain why $\mathrm{CC}$ occurs. Initially, the $\mathrm{CC}$ effect was attributed to improving the deployment of attention within a scene (Chun \& Jiang, 1998). It was thought that information within a repeated context would be able to "guide" your attention to the target so that you could find it faster. Although a context may facilitate guidance of attention, especially when participants have more time with a repeated display prior to response (Kunar, Flusberg, \& Wolfe, 2008b; see also Johnson, Woodman, Braun, \& Luck, 2007), we have proposed a second theory, which suggests that CC may speed the response process (Kunar, Flusberg, Horowitz, \& Wolfe, 2007; Schankin \& Schubö, 2009). In particular, the threshold needed to commit to a response in a repeated display may be reduced if the participant expects to see the target in a particular location within that context.

Regardless of the mechanism behind $\mathrm{CC}$, it is important to note that it is a robust and easily replicated effect: Repeating the context reliably facilitates target-present responses (e.g., Chun, 2000; Chun \& Jiang, 1998, 1999, 2003: Endo \& Takeda, 2004; Hoffmann \& Sebald, 2005; Jiang \& Chun, 2001; Jiang \& Leung, 2005; Jiang, Song, \& Rigas, 2005; Jiang \& Wagner, 2004; Kunar, Flusberg, Horowitz, \& Wolfe, 2007; Kunar, Flusberg, \& Wolfe, 2006; Lleras \& Von Mühlenen, 2004; Olson \& Chun, 2002; Tseng \& Li, 2004). Participants are certainly learning something about repeated displays, but what is it that they are learning? Recent work has suggested that, when the configuration of the repeated stimuli is invariant ("configural CC"), the relationship between the target and its surrounding distractors is what is learned. We know that learning the absolute positions of the target items alone is not enough (see Chun \& Jiang, 1998). Therefore, it must be the pattern of distractors or the stable relationship of the target to the distractors that drives the $\mathrm{CC}$ benefit.
In a further investigation of this point, Olson and Chun (2002) manipulated repeated target displays by having the repeated context occupy one side of the display, while the other side of the display was free to randomly change from trial to trial. In these studies, Olson and Chun found that a $\mathrm{CC}$ effect could still be obtained as long as the relationship between the target with its immediately surrounding distractor items (e.g., in the invariant half of the display) was preserved. Similarly, Jiang and Wagner (2004) found that when they recombined two old displays (e.g., Displays $\mathrm{A}$ and $\mathrm{B}$, which both predicted the target to be in the same location) to produce a "new" display, a full CC effect was found as long as some of the target-distractor associations were maintained (i.e., half of the distractors were taken from Display A and half from Display B). Finally, Brady and Chun (2007) found that a CC effect still emerged if distractors in only one quadrant of the display cued the target's location (i.e., only 2 nearby distractors out of 11 were predictive). The results showed that the $\mathrm{CC}$ effect observed in this "quadrant-predictive" condition was the same as that observed in a condition in which all of the distractors predicted the target location (providing that the quadrant did not move position around the screen). In essence, it seems that the benefit obtained from repeatedly seeing a context is derived from the relationship between the target and its immediately surrounding distractors.

Notice that within these studies the relationship between the target and distractors was the manipulated variable. What happens when the context tells the observer that the target is not present? On first glance, one might predict that people could use this contextual knowledge to facilitate a response when the target was absent. Imagine searching for a student in a crowded lecture theatre. From previous experience, you know that this particular student always sits in the front row-three spaces to the left of the aisle. When the student is there, you use this contextual knowledge to help find her. Intuitively, you would think that the same knowledge could speed your conclusion that she is absent from that lecture. If she is not sitting in that particular seat, chances are that she is not there at all. In fact, this pattern of data has been witnessed in the repeated search literature, in which participants search the same display again and again (Wolfe, Klempen, \& Dahlen, 2000; see also Kunar, Flusberg \& Wolfe, 2008a, and Oliva, Wolfe, \& Arsenio, 2004). Although search slopes in these experiments did not become more efficient, RTs became faster over time in both the target-present and target-absent displays (Wolfe et al., 2000; see also Kunar et al., 2007, for similar evidence in CC displays).

However, if the target-distractor associations are what is important in configural $\mathrm{CC}$, a predictive context may not necessarily facilitate RTs in target-absent trials. If there is no target-distractor association to be learned, in the absence of 
a target, then perhaps, counter to the analogy above, there will be no CC. In this study, we investigated what happens when the target is sometimes absent from the display. To anticipate our results, we found that $\mathrm{CC}$ is largely confined to target-present trials in displays in which the configuration acts as the context.

In Experiment 1, we investigated whether a $\mathrm{CC}$ effect is observed when, in half of the displays, the target is absent, and in the other half, the target is present. For example, in a display with Configuration $\mathrm{A}$, the target was always present at location $(x, y)$, while in a display with Configuration $\mathrm{B}$, the target was always absent. The results suggest that a CC effect only occurs in target-present displays. Experiment 2 investigated whether people can make use of the context when the same display predicts both target-present and target-absent responses [i.e., do people learn that in Display $\mathrm{A}$, if the target is not at location $(x, y)$, it is not there at all]? In this case, when the same context can accompany both target-present and target-absent trials, no CC benefit was observed on either target-present or -absent trials. In Experiments 3 and 4, the target was always present but could appear in multiple possible target locations. In Experiment 3, distractors appeared in any of the possible target locations that did not happen to contain the target, while in Experiment 4 distractors never appeared in those target locations. The results suggest that the appearance of a distractor in a previously designated target location is what disrupts CC. Experiment 5 showed that having a distractor appear in a target location disrupts the expression of CC, for both target-present and -absent displays, even when the context has previously been successfully learned. In this case, changing the response dimension from an orientation task to a present/absent task removes the CC effect. In the General Discussion, we discuss the results in relation to the attentional guidance and response selection accounts of CC.

\section{Experiment 1}

Previous experiments investigating $\mathrm{CC}$ have tended to use paradigms in which the target was always present (e.g., participants had to respond to whether a target $\mathrm{T}$ was facing to the left or the right; Chun \& Jiang, 1998; Kunar et al., 2007). We investigated whether a CC effect occurs when participants have to respond to the presence or absence of a target. In this experiment, one set of displays always contained a target, while in the other set of displays a target was never present.

Method

Participants A group of 13 naïve observers served as participants. All were recruited on the basis that they fell within the age range of $18-55$ years and had normal or corrected-to-normal vision. All participants gave informed consent and were paid for their time.

Apparatus and stimuli The experiment was conducted on a Macintosh computer using MATLAB software with the Psychophysics Toolbox (Brainard, 1997; Pelli, 1997). The distractor items were white $\mathrm{L}$ shapes presented randomly in one of four orientations $\left(0^{\circ}, 90^{\circ}, 180^{\circ}\right.$, or $\left.270^{\circ}\right)$. The target item (if present) was a white $\mathrm{T}$ shape rotated $90^{\circ}$ either to the left or to the right with equal probabilities. Each L contained a small offset (approximately $0.1^{\circ}$ ) at the line junction, to make search more difficult (see Fig. 1). All stimuli subtended $1.2^{\circ} \times 1.2^{\circ}$ at a viewing distance of $57.4 \mathrm{~cm}$ and were presented in an invisible $8 \times 12$ matrix that subtended $34.6^{\circ} \times 25.6^{\circ}$. The background was a uniform gray.

Procedure The experiment consisted of six epochs, in turn made up of five blocks apiece, each containing 24 trials. Within a block, 12 displays were repeated displays, in which the target and distractor locations remained the same throughout the experiment. These configurations were repeated once in each block throughout the experiment. The configurations of the other half of the trials were never repeated ("unrepeated" displays). In a CC experiment, it is important to assure that participants are learning the context of the display and not merely learning likely target locations. Accordingly, on the unrepeated trials, targets were restricted to 12 locations (see Chun \& Jiang, 1998). However, in these trials, the relationship of targets to distractors was not preserved from trial to trial.

On any given trial, the target item (if present) was presented along with 11 distractor Ls so that the total set size equaled 12. If the target was absent, 12 distractor Ls were presented. Half of the repeated displays were designated to always be target-present displays, while the other half were target-absent displays. Likewise, in the unrepeated displays, half of the trials were target present and the other half were target absent. Thus, a repeated configuration of items could either inform a participant about the location of a target or about the absence of any target in that particular configuration. Participants were
Fig. 1 Example display for Experiment 1

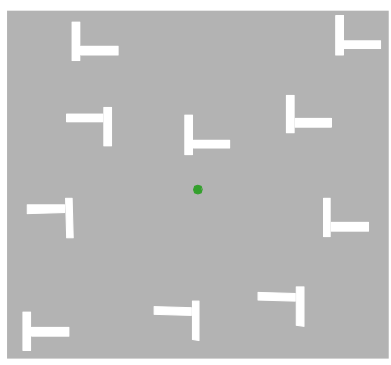


asked to search for the target, $\mathrm{T}$, and to press a right key ("l") if it was present or a left key ("a") if it was absent. They were asked to respond as quickly but as accurately as possible. Participants completed a practice block of 24 trials before the experiment proper. An example display can be seen in Fig. 1.

Data analysis In order to assess whether there was a CC effect or not, we measured the difference between repeated and unrepeated configurations across the last three epochs. Chun and Jiang (1998) first used this method, and we have also previously used it as a way of defining whether a given context benefits RTs (Kunar et al., 2007; Kunar et al., 2006; Kunar et al., 2008b). Although a CC effect can emerge early in the experiment (as mentioned above, it can be observed after five repetitions), in general, the more times a display is repeated, the greater the $\mathrm{CC}$ benefit.

\section{Results and discussion}

One participant was removed from the analysis due to high error rates $(38 \%)$. In the remaining data, fewer errors were made when the target was absent $[F(1,11)=29.2, p<.01]$, and marginally fewer errors were made when the target was in a repeated display $[F(1,11)=4.8, p=.051]$. The interaction was not significant. Because the error rates were low (6.6\%) and we were more concerned with RTs, we do not discuss errors further.

Here, and in all subsequent experiments, RTs below $200 \mathrm{~ms}$ and above 4,000 ms were removed. An overall ANOVA with the factors Presence (target present or target absent), Display Type (repeated or unrepeated), and Epoch (1-6) on RTs showed a main effect of presence, $F(1,11)=$ $88.1, p<.01$, in which RTs were faster for target-present than for target-absent trials, and a main effect of epoch, $F(5,55)=26.6, p<.01$, in which RTs decreased across epochs. The Configuration $\mathrm{x}$ Presence interaction was marginally significant, $F(1,11)=4.0, p=.07$, and the Presence $\times$ Epoch interaction was significant, $F(5,55)=10.9, p<.01$. Repeated RTs were faster than unrepeated RTs in targetpresent but not in target-absent trials, and RTs decreased more across epochs in absent than in present trials. None of the other interactions were significant.

Because our main question of interest was to investigate the separate CC effects for both target-present and targetabsent displays, two subsequent $2 \times 2$ ANOVAs (with the factors Configuration and Epoch) were conducted on present and absent RTs, respectively (see Fig. 2). Let us examine the present trials first. RTs from repeated displays were faster than those for unrepeated displays, $F(1,11)=5.1$, $p<.05$, and overall RTs decreased over epochs, $F(5,55)=$ $16.48, p<.01$. There was no interaction between Display
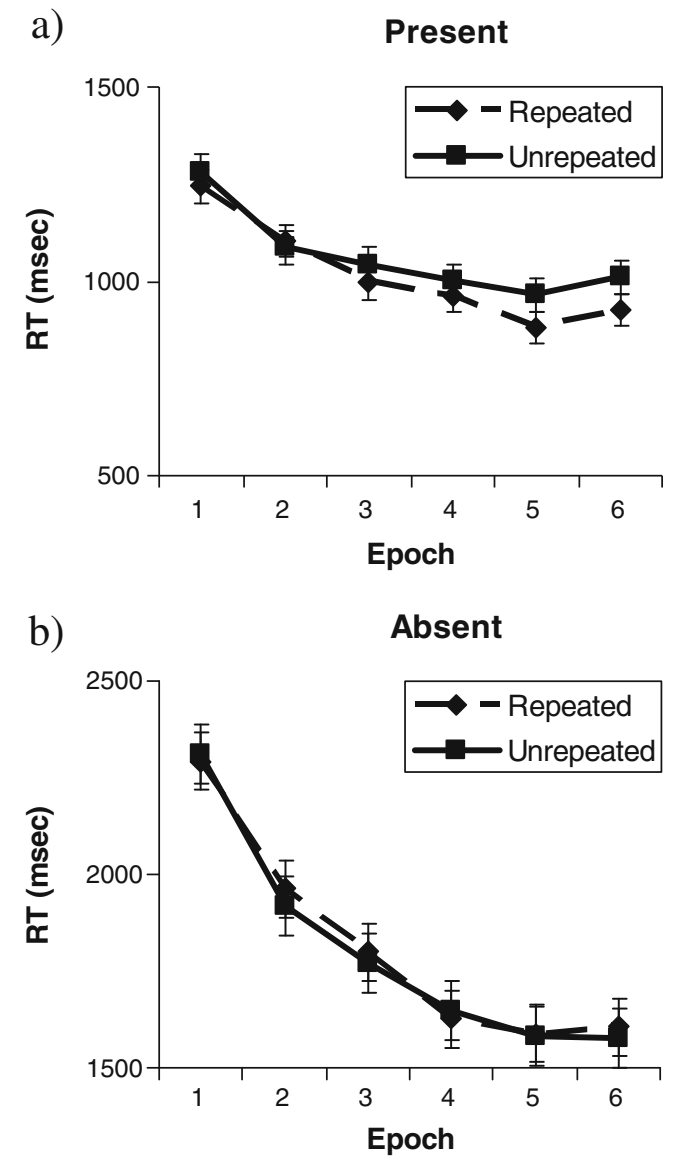

Fig. 2 Mean correct RTs (in milliseconds) across epochs for targetpresent and target-absent trials in Experiment 1. Please note that although there are different $y$-axis values on the target-present and target-absent graphs, the relative scale ranges of $1,000 \mathrm{~ms}$ are equivalent

Type and Epoch $(F<1)$. Taking our standard measure of CC, however (i.e., the difference between RTs in repeated and unrepeated displays over the last three epochs), we see that a $\mathrm{CC}$ effect occurred for target-present displays: Participants were $70 \mathrm{~ms}$ faster at responding in repeated than in unrepeated displays, $t(11)=-2.6, p<.05$.

In contrast, there was no benefit of repeating the context in target-absent displays. Although RTs generally decreased across epochs, $F(5,55)=23.9, p<.01$, there was no main effect of display type, nor a significant Display Type $\times$ Epoch interaction. Examining data from the last three epochs, there was no evidence of a CC effect, $t(11)=0.2, \mathrm{n}$. $\mathrm{s}$. If anything, participants were $5 \mathrm{~ms}$ slower at responding to repeated than to unrepeated displays.

In this experiment, although there was a $\mathrm{CC}$ effect in target-present trials, there was no CC effect when the target was absent. This fits with the hypothesis that the context is encoded relative to a target item (e.g., Brady \& Chun, 2007) and argues against the notion that the context could inform an observer about the absence of a target. 


\section{Experiment 2}

If a context was only associated with the absence of a target, CC was not observed. What happens when a spatial context specifies the location of a target, if that target is present, but where it is possible for the target to be absent on a given trial? There are four possible outcomes. First, a $\mathrm{CC}$ effect could occur in both target-present and targetabsent displays. Participants may learn that in a given configuration the target will only appear at one location. If the target is not at that location, then it is not there at all. Participants could use this information to speed both targetpresent and target-absent responses to repeated contexts. This would be the most obvious prediction of a model that argues that context guides attention to the target location. Second, a CC effect could occur in target-present displays but not in target-absent displays, as in Experiment 1. Third, a CC effect could occur in target-absent displays but not in target-present displays. Although this is logically possible, prior work has made it seem unlikely. In fact, in Experiment 2 we found that the answer is the fourth logical option: CC effects do not occur in either target-present or target-absent displays. Having a distractor appear in the place of the target seems to disrupt learning of the target-distractor associations, rendering them "unreliable." A high degree of reliability may be required to produce a $\mathrm{CC}$ speeding of responses to targets (see also Jungé, Scholl, \& Chun, 2007).

\section{Method}

In Experiment 1, the target was present on $50 \%$ of trials. There were two versions of Experiment 2. In one, the target was present $50 \%$ of the time, and in the other, the target was present $75 \%$ of the time. For the remainder of the trials, the target was absent. In absent trials, a distractor item appeared at the "target" location.

Participants A group of 13 naïve observers between the ages of 18 and 55 years served as participants for the $50 \%$ condition, and 12 naïve observers served as participants for the $75 \%$ condition. All participants were recruited to fall within the age range of $18-55$ and to have normal or corrected-to-normal vision. All participants gave informed consent and were paid for their time.

Apparatus and stimuli The apparatus and stimuli were identical to those of Experiment 1.

Procedure The procedure was similar to that of Experiment 1 , except that here the target could be either present or absent when a repeated context was shown. For any repeated display, targets appeared on average $50 \%$ of the time in the $50 \%$ condition, and $75 \%$ of the time in the $75 \%$ condition. On the other trials, the target was absent and the "target location" was filled with a distractor. For unrepeated displays, again the potential target locations were filled with targets on $50 \%$ of the trials in the $50 \%$ condition and $75 \%$ of the trials in the $75 \%$ condition. On the remaining trials, the target was absent and its location was filled with a distractor. In the $75 \%$ condition, the size of the stimuli varied depending on their eccentricity (since visual acuity declines as a function of the distance from the center point). Those closest to the center subtended $1^{\circ}$ of visual angle, while those farther away subtended $1.5^{\circ}$ or $2.5^{\circ}$, depending on their relative distance from the center of the display. The horizontal and vertical lines of the $\mathrm{L}$ also made a perfect right angle and were not offset. Please note that these small changes do not affect the overall CC effect (see Kunar et al., 2007, who used these stimuli) - though, as can be seen in the faster RTs of Fig. 3, these simpler stimuli made the task somewhat easier.

For the $50 \%$ condition, the numbers of trials and epochs were similar to those in Experiment 1. For the 75\% condition, there were 448 experimental trials, divided into seven epochs of 64 trials each. Overall, there were four repeated displays, each repeated eight times per epoch, and the other half of displays were unrepeated. Participants were instructed to press the letter " $m$ " when the target $T$ was present and the letter " $z$ " when it was absent. Although the numbers of repeated and unrepeated displays were decreased in this condition, the number of repetitions per display increased. Previous research has shown that this increase in repetitions has led to a robust (and numerically large) CC effect (Kunar et al., 2007; Kunar et al., 2006, 2008b; see also the training phase in Exp. 5). If a CC effect does occur when the target is sometimes absent, one would suggest that with these conditions, a CC effect should emerge.

\section{Results and discussion}

As can be seen in Fig. 3, the main result of both versions of Experiment 2 was that $\mathrm{CC}$ was eliminated, even for the target-present trials.

There were more errors on target-present than on targetabsent trials, $F(1,12)=71.2, p<.01$, in the $50 \%$ condition, but not in the $75 \%$ condition. None of the other main effects or interactions were significant. Overall, error rates were low in both the $50 \%$ and $75 \%$ conditions $(8 \%$ and $5 \%$ ), and so are not discussed further.

An overall ANOVA with the factors Presence, Display Type, and Epoch on RTs for each condition ${ }^{1}$ showed there

\footnotetext{
${ }^{1}$ Because the $50 \%$ condition and the $75 \%$ condition had different epochs, it was not viable to conduct an overall between-conditions ANOVA on the two conditions.
} 
Fig. 3 Mean correct RTs (in milliseconds) across epochs for target-present and target-absent trials in Experiment 2
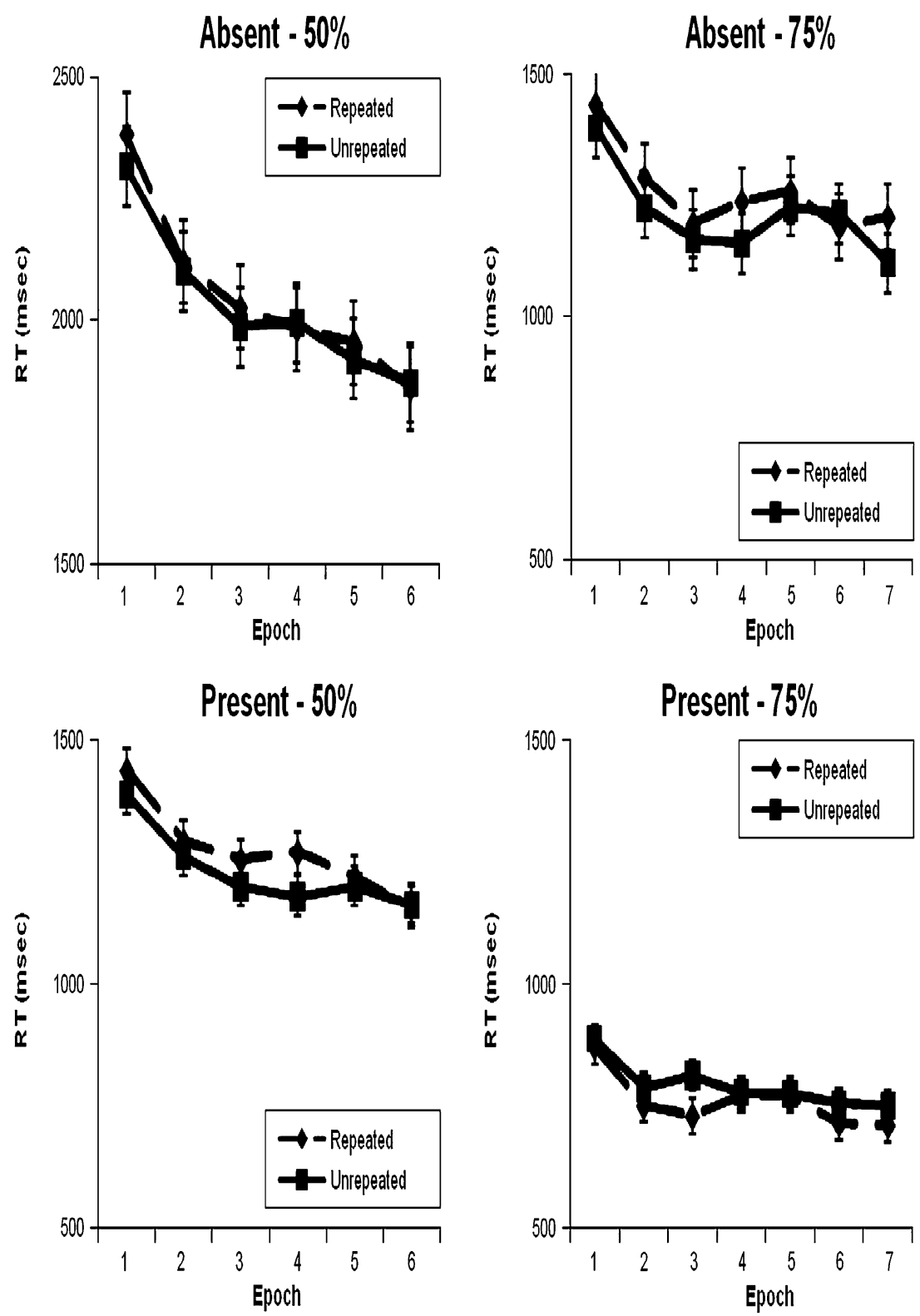

to be a main effect of presence- $F(1,12)=48.8, p<.01$, and $F(1,11)=26.0, p<.01$, for the $50 \%$ and $75 \%$ conditions, respectively-where RTs were faster for targetpresent than for target-absent trials, and a main effect of epoch $-F(5,60)=16.9, p<.01$, and $F(6,66)=6.4, p<$ .01 , for the $50 \%$ and $75 \%$ conditions, respectively-where RTs decreased across epochs. The Configuration x Presence interaction was marginally significant, $F(1,11)=3.5, p=.09$, and significant $F(1,11)=5.0, p<.05$, in the $50 \%$ and $75 \%$ conditions, respectively, and there was a significant Presence $\mathrm{x}$ Epoch interaction in the $50 \%$ condition, $F(5,60)=6.6, p<$ .01 . None of the other interactions were significant.

Because we were interested in the $\mathrm{CC}$ effects for targetpresent and target-absent trials, the data were split up into individual $2 \times 2$ ANOVAs with the factors Display Type and
Epoch for each condition. Figure 3 shows RTs for both targetpresent and target-absent trials for both conditions. As can be seen, none of the target-present or target-absent trials showed a $\mathrm{CC}$ effect. In fact, on target-present trials in the $50 \%$ condition, RTs from repeated displays were actually slower than those for unrepeated displays, $F(1,12)=14.7, p<.01$. Overall, RTs decreased over epochs in both conditions, $F(5,60)=8.3, p<$ .01 , and $F(6,66)=5.0, p<.01$, for the $50 \%$ and $75 \%$ conditions, respectively. There was no interaction between display type and epoch in either condition. Looking at our standard measure of $\mathrm{CC}$, we see that, unlike in Experiment 1, there was no $\mathrm{CC}$ effect in either the $50 \%$ or the $75 \%$ condition, $t(12)=1.5$, n.s., and $t(11)=-1.3$, n.s., respectively.

In target-absent trials, RTs from repeated displays were marginally slower than those for unrepeated dis- 
plays, $F(1,12)=3.7, p=.08$, in the $50 \%$ condition, and significantly slower in the $75 \%$ condition, $F(1,11)=5.0$, $p<.05$. Overall, RTs decreased over epochs, $F(5,60)=18.4$, $p<.01$, and $F(6,66)=3.8, p<.01$, for the $50 \%$ and $75 \%$ conditions, respectively. There was no interaction between display type and epoch in either condition. Again, using the standard measure of $\mathrm{CC}$, there was no $\mathrm{CC}$ effect in either the $50 \%$ or the $75 \%$ condition, $t(12)=0.1$, n.s., and $t(11)=1.4$, n.s., respectively.

Having a display be both "target present" and "target absent" seems to prevent the development of the CC effect. Using the standard measure of CC, there was no benefit of having a predictive context. Indeed, the effect of context went the other way. A replication of this study, using set sizes of both 8 and 12, failed to find a main effect of display type at either set size. Again, in this replication, RTs for target-present repeated displays were marginally slower than those for unrepeated displays, $F(1,7)=4.46, p=.07$. Put together, the data suggest that replacing a target with a distractor on target-absent trials eliminates CC or, perhaps, even creates a modest "contextual confusion" on the targetpresent trials.

Makovski and Jiang (2010) recently showed evidence of a contextual cost (in which RTs for repeated displays were slower than those for unrepeated displays) under some conditions. In their experiments, they manipulated the $\mathrm{CC}$ effect observed when the target item systematically moved farther away, in the test phase, from its location learned in the training phase. In their Experiment 1, they moved the target to an empty location, while in Experiment 2 they swapped the location of the target and a distractor, so that a distractor appeared in the previous target location. In the latter experiment, a small CC cost emerged. Makovski and Jiang attributed this cost to the distance that the target had moved. However, in light of the present results, we suggest that the contextual cost may have occurred because of the presence of a distractor appearing in a target location.

\section{Experiment 3}

Experiment 2 showed that when the target was replaced with a distractor item in target-absent trials, no CC effect was found in either present or absent trials. The root cause of this failure may have been disruption of the normal $\mathrm{CC}$ effect by the presence of a distractor at the target location. Would the presence of a distractor at a target location continue to be disruptive if targets were present on every trial? We investigated this in Experiment 3, in which the target was always present but could appear at a number of predesignated locations. As we will see, the presence of a distractor in a target location was enough to disrupt CC. No $\mathrm{CC}$ effect was observed when the target could be found in more than one location in a repeated display — at least, not if distractors filled the other locations.

\section{Method}

In Experiment 3, a single target was present on each trial. Within a given repeated configuration, targets could appear in one of one, two, three, or four possible locations. If a location was not occupied by a target item, it was filled by a distractor. The configuration was $100 \%$ predictive only when there was one possible target location. Participants could, in principle, learn that the target would always be at either Location A or Location B when there were two locations, and so forth for three or four locations.

Participants A group of 16 naïve observers served as participants. All fell within the age range of 18-55 years and had normal or corrected-to-normal vision. All participants gave informed consent and were paid for their time.

Apparatus and stimuli The apparatus and stimuli were identical to those of Experiment 1.

Procedure Participants were asked to search for the target, T, and to press a left key ("a") if it was rotated to the left and a right key ("l") if it was rotated to the right. The target was always present. They were asked to respond as quickly and accurately as possible. On any given trial, the target item was presented along with 11 distractor Ls, so that the total set size equaled 12 . The experiment consisted of 120 blocks, each containing 24 trials. Within a block, half of the trials had a repeated configuration. These configurations were repeated once in each block throughout the experiment. The other half of the trials were the unrepeated displays. Of the 12 repeated configurations, 3 had a single target location (akin to the standard CC paradigm), 3 had two possible locations, 3 had three possible locations, and 3 had four possible locations. Since there was only one target on any given trial, the other possible target locations contained a distractor. This preserved the exact spatial configuration for the repeated displays. The same rule applied to the choice of target locations for the unrepeated configurations. Of the 12 unrepeated configurations, 3 were constrained so that the target only appeared in one location, 3 so that the target could appear in two possible locations, 3 so that the target could appear in three possible locations, and 3 so that the target could appear in four possible locations. Any simple location priming would be the same in repeated and unrepeated conditions. However, there could be no $\mathrm{CC}$ in the unrepeated conditions, since the configuration of the rest of the display was not held constant.

As in Experiments 1, for the purposes of analysis, blocks were grouped in sets of five, to make 24 epochs. In the 
standard CC experiments of Chun and Jiang, there were only six epochs, and each display was presented 30 times (see, e.g., Chun \& Jiang, 1998). Because we increased the number of possible target locations within a configuration from one to four, we also increased the number of epochs (and, hence, display repetition) fourfold. This ensured that, by the end of the experiment, every possible display combination had been presented at least 30 times (comparable to previous $\mathrm{CC}$ studies and to Exps. 1 and 2 here). Because the experiment took approximately $4 \mathrm{~h}$ to complete, it was broken down into two sessions, with the constraint that each session was run within a week of the other (since it has been found that successful CC effects persist for the time span of a week; Chun \& Jiang, 2003).

Participants completed a practice block of 24 trials before the experiment proper. At the end of the experiment, they were asked whether they had noticed that some of the configurations were repeated and to identify which of the configurations they thought they had seen before. If they were unsure, they were asked to guess. Example displays are shown in Fig. 4.

\section{Results and discussion}

Overall, error rates were quite low, at $4 \%$ in both repeated and unrepeated displays, with no significant effects of display type or number of target locations. Nor was the interaction reliable. As such, we do not discuss errors further. Eight of the participants reported having recognized that some of the configurations were repeated. However, when asked to explicitly report which configurations they had seen, all of them failed to do so correctly. The overall accuracy for configuration identification equaled $40 \%$ (with a chance level of $50 \%$ ), with participants who reported explicit recognition averaging only $36 \%$ correct.

In all the following analyses, RTs from repeated displays were compared to those of the unrepeated displays with the same numbers of absolute target locations. Figure 5 shows RTs for repeated and unrepeated configurations, as a function of the number of potential target locations. As is clear from the figure, there was a convincing $\mathrm{CC}$ effect only

A

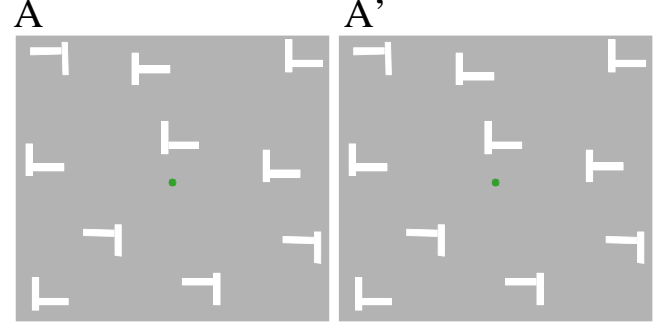

Fig. 4 Example displays for repeated trials in Experiment 3. Panels A and $\mathrm{A}^{\prime}$ have the same repeated configuration. However, a distractor in Display $\mathrm{A}^{\prime}$ occupies the previous target location in Display A when there was just one possible target location. Overall, RTs became faster over time, $F(23,345)=27.8, p<.01$, and although there was no main effect of display type, $F(1,15)=1.6$, n.s., there was a reliable Display Type $\times$ Epoch interaction, $F(23,345)=1.7, p<.05$ : RTs from repeated displays became faster across epochs relative to RTs for unrepeated displays. Looking at the standard measure of $\mathrm{CC}$, we see that RTs for repeated displays were marginally faster than those for unrepeated displays, $t(15)=-2.0, p=.06$.

When there were two, three, or four possible target positions, none of the main effects of display type nor the Display Type $\mathrm{x}$ Epoch interactions approached significance. Looking at the standard measure of CC, we see that no CC effect was found when there were two or three possible target positions (all $t \mathrm{~s}<1$ ). However, repeated RTs were faster than unrepeated displays when there were four possible target positions, $t(15)=-4.2, p<.01$.

Examining our data, we see that not every participant showed a CC effect with one target location (and, in fact, prior work has suggested that not all participants show a CC effect under standard conditions; Lleras \& Von Mühlenen, 2004). Perhaps CC for displays with multiple target locations would be seen in the subset of observers who show the basic effect for a single location. Accordingly, we divided participants into two subsets: those who showed a CC effect with one target and those who did not (this was done by finding the difference in RTs between unrepeated and repeated configurations across Epochs 4-6 for each participant; see Chun \& Jiang, 1998). Participants who were allocated to the $\mathrm{CC}$ group showed a positive overall $\mathrm{CC}$ effect [i.e., for this group, the difference between unrepeated and repeated RTs was greater than zero, $t(10)=3.1, p=.01$ ], whereas participants who were allocated to the no-CC group showed a negative effect [i.e., for this group, the difference between the unrepeated and repeated RTs was less than zero, $t(10)=3.8, p=.02]$. Five of the participants (29\%) did not show a CC effect, so their data were excluded from further analysis. Figure 6 shows RTs, for the CC group only, for repeated and unrepeated displays as a function of on the number of potential target locations.

Unsurprisingly, since they were selected on this basis, these observers showed a CC effect when there was only one possible target location. Overall, the observers were faster at finding a target if it was embedded in a repeated configuration than if it was in an unrepeated configuration, $F(1,10)=7.6, p<.05$. Likewise, RTs became faster across epochs, $F(23,230)=20.0, p<.01$. The Configuration $\times$ Epoch interaction did not prove reliable, $F(23,230)=1.3$, n.s. Looking at the standard measure of $\mathrm{CC}$, repeated RTs were faster than unrepeated RTs, $t(10)=-3.4, p<.01$.

Of more interest, this was not the pattern of results when there were two, three, or four potential target locations. 
Fig. 5 Mean correct RTs (in milliseconds) for each condition in Experiment 3: trials that had one, two, three, or four possible target locations. Please note that error bars, for the most part, are occluded by the symbols
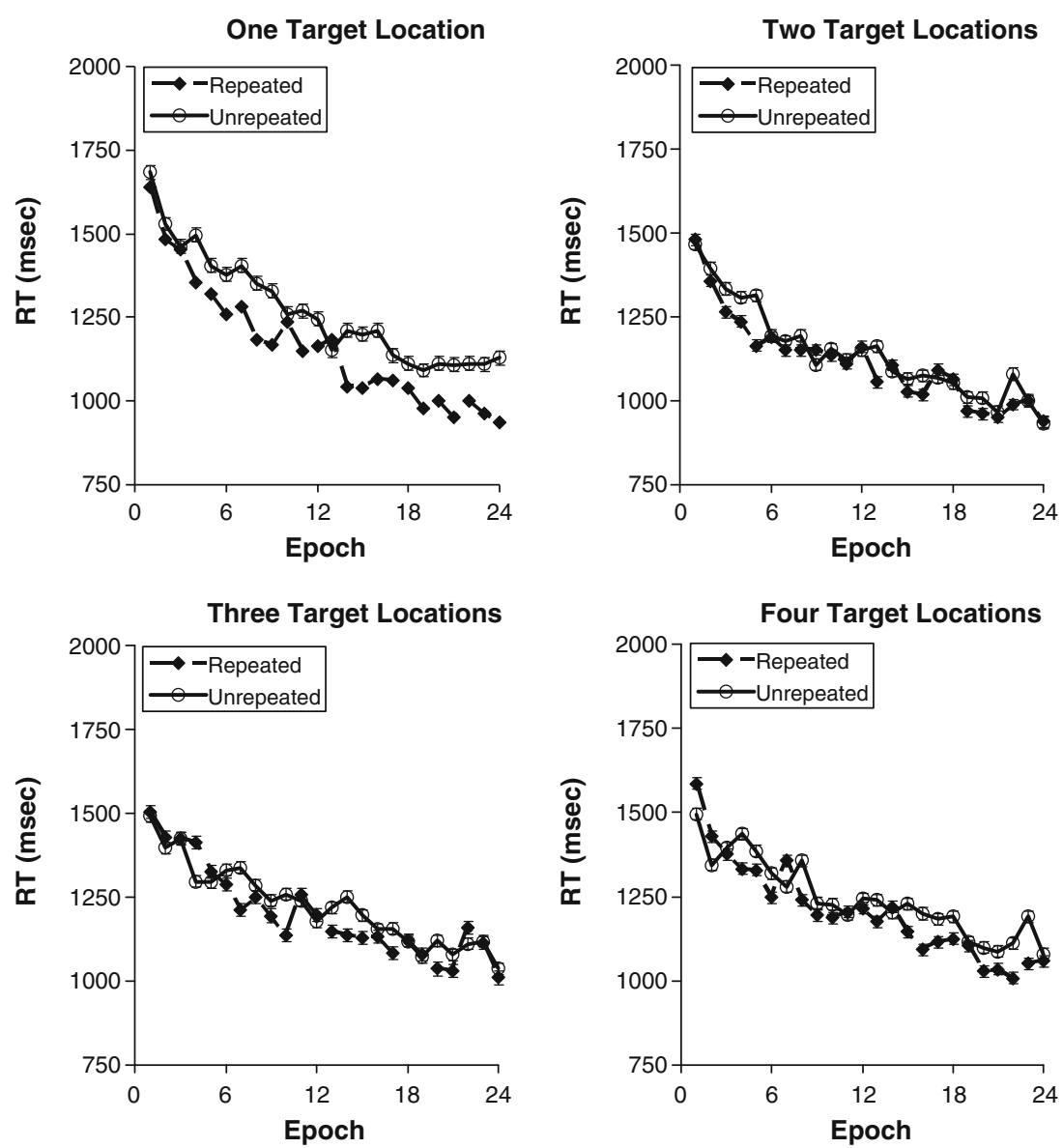

Overall, RTs for two, three, or four potential targets became faster over epochs (all $F_{\mathrm{s}}>8.9, p \mathrm{~s}<.01$ ). However, the main effects of display type failed to reach significance for two, three, or four target locations (all $F_{\mathrm{s}}<2.2$, all $p \mathrm{~s}>$ .17). RTs for repeated configurations did not differ from those for unrepeated configurations. If we examine Fig. 6 closely, there is the barest hint of an effect; repeated RTs are slightly faster than unrepeated RTs for most epochs, especially, it seems, in the three-target case. This gains some statistical support, in the form of reliable Display Type $\mathrm{x}$ Epoch interactions for three and four potential target locations $-F(23,230)=2.1, p<.01$, and $F(23,230)=1.6$, $p<.05$, respectively - but not for two target locations, $F(23,230)=0.7$, n.s. Data from the last three epochs (the standard measure of CC) showed that there was no difference between repeated and unrepeated RTs when there were either two or three target locations (all $t \mathrm{~s}<1$ ). When there were four target locations, repeated RTs were on the whole faster than unrepeated RTs, $t(10)=-2.5, p<.05$. Despite this, examining the figures, it is clear that any CC effect is very weak and clearly different from the case of one target location. Furthermore, any $\mathrm{CC}$ effect is not consistent across the latter epochs (e.g., the planned comparisons between repeated and unrepeated configurations for the last epoch do not prove reliable, as would be predicted if a strong CC effect were present; all $t \mathrm{~s}<1.4, p \mathrm{~s}>.2$ ). We would be hard pressed to claim a strong effect of repeating the display in any of the displays except those with one target location.

A standard CC effect was observed when the configuration predicted only one target location. However, if the configuration predicted more than one target location, with a distractor in any previous target location, there was very little benefit of CC. This negative finding could have occurred for a number of reasons. First, it could reflect a lack of statistical power; maybe the effect is present but too small to be seen reliably. This seems unlikely. The number of specific pairings of a context and a target location was equal to or greater than that used in a standard CC experiment-even when the target could appear in one of four locations. If a standard CC effect were present with multiple target locations, this experiment should have produced it in all conditions. More plausibly, it could be that people do not show $\mathrm{CC}$ if a distractor sometimes appears in a potential target location (see also Exp. 2), because that disrupts the learning of the target-distractor associations. If the target is not always in a specific location, the observers might not utilize the contextual 
Fig. 6 Mean correct RTs (in milliseconds) for each condition in Experiment 3, only for participants who showed a valid contextual cuing effect with one target location. The different panels show RTs for trials that had one, two, three, or four possible target locations. Please note that error bars, for the most part, are occluded by the symbols
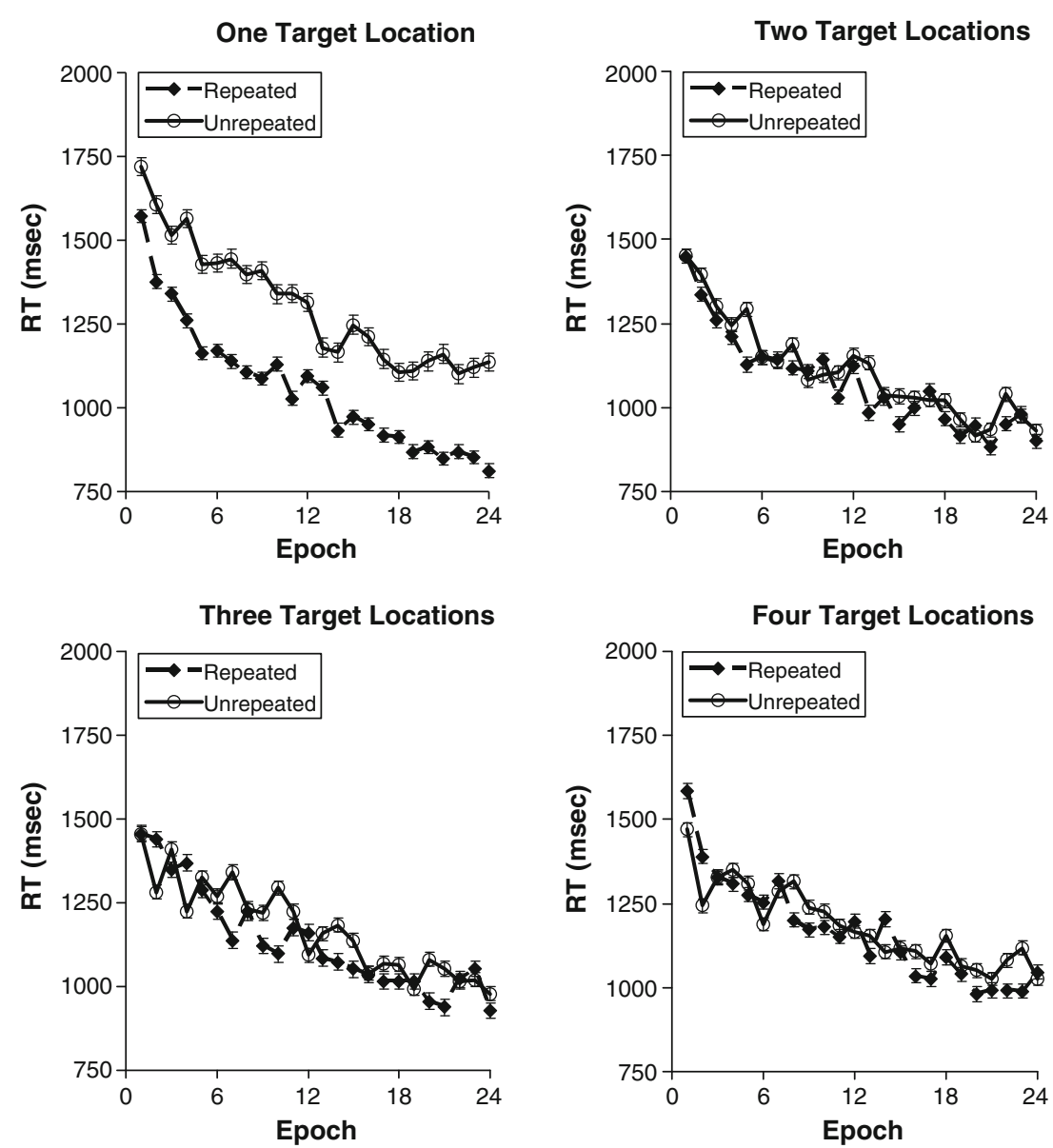

information, and instead might search the display as if it had not been seen before, without the memory of any display regularities (see also Kunar, Flusberg, \& Wolfe, 2008a; Oliva et al., 2004; Wolfe et al., 2000).

Given that there was a statistical Display Type $\times$ Epoch interaction for the three- and four-target location conditions, one could argue that maybe $\mathrm{CC}$ was present in these displays, but greatly reduced. This is plausible, but it again points to the fact that having a distractor in a target location interferes with the $\mathrm{CC}$ effect so that it is reduced at the very least, if not removed entirely. Other work has shown that CC can occur for multiple target locations. Chun and Jiang (1998) showed that up to two target locations can be cued by the same configuration. The critical difference between their work and our work presented here was that, in Chun and Jiang's (1998) study, the two potential target locations always remained unoccupied if the target did not appear at that position. A distractor never appeared in a target position. In our work, however, when the target was not present at one of the potential target locations, a distractor occupied that place. This seems to be a key factor in establishing whether or not a CC effect will be observed, and was investigated further in Experiment 4.

\section{Experiment 4}

Experiment 3 suggested that the presence of a distractor item appearing in a target location disrupts CC. If this is the case, then a $\mathrm{CC}$ effect might reemerge when there are multiple possible target locations but when a distractor never appears in a target location. This was investigated in Experiment 4.

\section{Method}

Participants A group of 12 naïve observers between the ages of 18 and 55 years served as participants. All had normal or corrected-to-normal vision. All participants gave informed consent and were paid for their time.

Apparatus and stimuli The apparatus and stimuli were identical to those of Experiment 1, except that here all of the stimuli were presented within square, white, outline boxes ("placeholders"), which marked out the configuration of a display. All placeholders subtended visual angles of $1.7^{\circ} \times 1.7^{\circ}$.

Procedure Participants were asked to respond to the orientation of the letter $\mathrm{T}$ as quickly and as accurately as 
possible. The use of 12 placeholders per configuration ensured that the overall shape and layout of the configuration was kept constant, regardless of target location (see Kunar et al., 2007, and Kunar et al., 2008b, who also used placeholders to mark out a repeated configuration). Items could only appear within a placeholder. Each configuration had four possible target locations. Since the number of placeholders was fixed at 12 and there were four potential target locations, eight distractor items were presented alongside the target. Thus, the set size was nine items (Ts and Ls) or 12 placeholders. As in the four-target condition of Experiment 3, in the unrepeated trials, the target position was limited to four possible locations, but the overall configurations of these displays varied across the experiment. However, unlike in Experiment 3, a distractor item never occupied a possible target location. There were three repeated configurations and three unrepeated configurations. In each block, each of the 3 repeated configurations was repeated four times (once with each possible target location) and was intermixed with 12 unrepeated configurations, to make a total of 24 trials per block. There were 30 blocks in total, so that each configuration-target combination was presented on the whole 30 times (with the overall placeholder configuration being repeated 120 times over 720 trials in total). Example displays are shown in Fig. 7.

\section{Results and discussion}

RTs for repeated and unrepeated configurations are presented in Fig. 8. It is clear that this condition produces CC. Overall error rates were low, at $1.4 \%$ in both old and new conditions, with no significant effects of configuration or epoch, or a significant Configuration $\mathrm{x}$ Epoch interaction. RTs from old configurations were faster than those from new ones, $F(1,11)=14.1, p<.01$, and RTs became faster across epochs, $F(5,55)=7.3, p<.01$. The Configuration $\times$ Epoch interaction did not prove reliable, $F(5,55)=1.8$, n.s. However, looking at the standard measure of $\mathrm{CC}$, there was a reliable

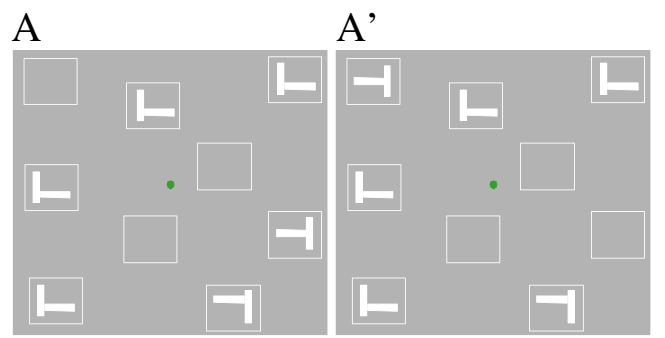

Fig. 7 Example displays for repeated trials in Experiment 4. Panels A and $\mathrm{A}^{\prime}$ have the same placeholder repeated configuration. However, the target in Display $\mathrm{A}^{\prime}$ occupies a different placeholder than that in Display A. In this experiment, a distractor never occupied a potential target location

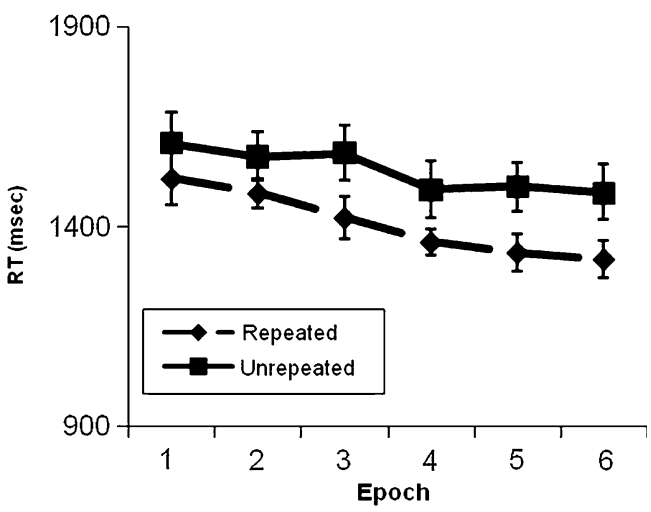

Fig. 8 Mean correct RTs (in milliseconds) across epochs in Experiment 4

effect of configuration, $t(12)=3.9, p<.01$. RTs became faster for repeated than unrepeated configurations over time.

Chun and Jiang (1998) found CC for two possible target locations. Experiment 4 extended that result to show CC for four target locations, as long as a distractor does not appear in a target location. These results clearly differ from those of Experiment 3, where any CC effect was minimal. It seems that having distractors appear in possible target positions weakens the $\mathrm{CC}$ effect. There are two possible reasons for this. First, having a distractor present in a target location might prevent learning of the repeated context by disrupting the target-distractor associations. Second, having a distractor in a target location might prevent the expression of contextual knowledge. We investigated this question in Experiment 5 by training in a standard CC condition in which observers learned the target-distractor associations in blocks of trials in which the target was always present. In the test phase, we examined whether participants could express this CC knowledge when a target could be either present or absent in the same display (as in Exp. 2).

To anticipate the results, Experiment 5 replicated the findings of Experiment 2. The results showed that CC does not occur on target-absent trials, even in conditions in which participants have successfully learned the context. Furthermore, even for a previously learned $\mathrm{CC}$ configuration, there was no $\mathrm{CC}$ effect on target-present trials if a distractor sometimes appeared in a target location. Target-absent trials on which a distractor was placed in the target location prevented the expression of even previously learned knowledge.

\section{Experiment 5}

Method

Participants A group of 24 naïve observers served as participants. All fell within the age range of $18-55$ years 
and had normal or corrected-to-normal vision. All participants gave informed consent and were paid for their time.

Apparatus and stimuli The apparatus and stimuli were similar to those of Experiment 1, except that here the experiments were programmed using Blitz Basic and run on PCs. In this experiment, all of the stimuli subtended visual angles of $1.7^{\circ} \times 1.7^{\circ}$ at a viewing distance of $57.4 \mathrm{~cm}$.

Procedure The experiment consisted of 32 practice trials and 576 experimental trials. In the experiment proper, participants were first given 448 "training" trials. In this phase, participants completed a standard CC task (in which half of the displays were repeated and the other half were unrepeated), similar to the target-present trials of Experiment 1. There were four repeated displays, each repeated eight times per epoch, and the other half of displays were unrepeated. There was always a target, $\mathrm{T}$, present in each display; participants pressed the letter " $\mathrm{m}$ " if the bottom of the T faced toward the right, and the letter " $\mathrm{z}$ " if the bottom of the $\mathrm{T}$ faced toward the left. The training phase was divided into seven epochs of 64 trials each, allowing participants to successfully learn the display contexts.

After the training phase, participants were then given the "test" phase, consisting of 128 trials (64 trials in Epoch 8 and 64 in Epoch 9). The test phase was similar to the training phase, except that in each repeated and unrepeated display, half of the time the target was present, and half of the time the target was absent (similar to the procedure of Exp. 2). Participants were asked to respond to the presence or absence of the $\mathrm{T}$ by pressing the letter " $\mathrm{r}$ " if the target was present or " $v$ " if the target was absent.

\section{Results and discussion}

As can be seen in Fig. 9, the introduction of target-absent trials in the test phase disrupted the $\mathrm{CC}$ that had developed in the training phase.

Overall, errors were low, at 3.6\%. The main effect of display type was not significant in the training phase. In the test phase, fewer errors were made in target-absent than in target-present trials, $F(1,23)=18.2, p<.01$, and fewer errors were made in repeated than in unrepeated trials, $F(1,23)=5.8, p<.05$. None of the interactions for the test phase were significant. We do not discuss errors further.

As expected, a CC effect developed during the training phase, indicating that participants successfully learned repeated contexts (see Fig. 9, "Training Phase"). A $2 \times 2$ ANOVA with the factors Display Type and Epoch on the training phase RTs showed that RTs were faster for repeated contexts than for unrepeated contexts, $F(1,23)=6.6, p<$ .05 , and RTs decreased over epochs, $F(6,138)=47.8, p<$ .01 . Although the Display Type $\times$ Epoch interaction was not
Training Phase

Test Phase

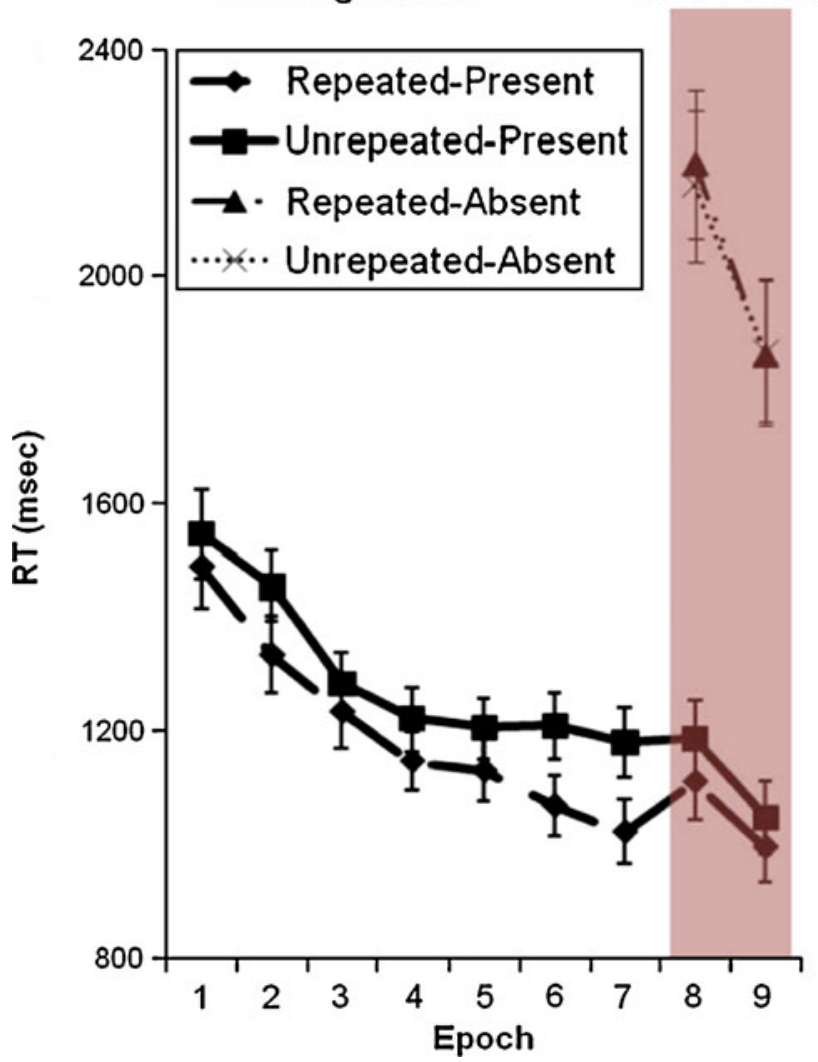

Fig. 9 Mean correct RTs (in milliseconds) across epochs for targetpresent trials in the training phase (Epochs 1-7) and for target-present and -absent trials in the test phase (Epochs 8-9) in Experiment 5

reliable, the standard measure of $\mathrm{CC}$ was significant: Repeated RTs were faster than unrepeated RTs over the last three epochs, $t(23)=-4.2, p<.01$.

While the training phase established successful contextual learning, the test phase was used to examine whether this learned knowledge could be expressed in situations in which the target was sometimes absent. An overall ANOVA with the factors Presence (target present and target absent), Display Type (repeated and unrepeated), and Epoch (8 and 9) on RTs in the test phase showed there to be a main effect of presence, $F(1,23)=122.3, p<.01$, with faster RTs in target-present trials than in target-absent trials, and a main effect of epoch, $F(1,23)=32.2, p<.01$, with RTs decreasing over epochs. There was also a Presence $\times$ Epoch interaction, $F(1,23)=9.1, p<.01$, with RTs decreasing more across epochs in the target-absent than in the target-present trials. None of the other interactions were significant.

To examine the potential $\mathrm{CC}$ effects for target-present and target-absent trials individually, the data were split up into two $2 \times 2$ ANOVAs with the factors Display Type and Epoch for target-present and target-absent trials, respectively. The results suggest that although people had learned the context in the training phase, the appearance of distractors 
in target locations during the test phase disrupted the CC effect on target-present trials (see Fig. 9, "Test Phase"). As before, there was no $\mathrm{CC}$ for target-absent trials. For target-present trials, although there was an effect of epoch, $F(1,23)=16.3, p<.01$, there was no main effect of context, $F(1,23)=2.8$, n.s. Nor was there a significant Context $\times$ Epoch interaction, $F(1,23)=0.2$, n.s. Having a distractor appear in a target location appears to have disrupted the expression of contextual knowledge, even when the context had been previously learned. We return to this point in the General Discussion. For target-absent trials, as in the previous experiments, $\mathrm{CC}$ was not seen. Although there was an effect of epoch, $F(1,23)=24.8, p<.01$, there was no effect of context, $F(1,23)=0.6$, n.s., nor was there a significant Context $\times$ Epoch interaction, $F(1,23)=1.1$, n.s.

Perhaps one reason why there was no observed CC effect in the test phase was that any benefit of having learned the context was masked by a task switch in the response keys pressed during the test phase. This might explain why there was an increase in RTs at Epoch 8. If so, one could argue that a $\mathrm{CC}$ effect would emerge if participants had undergone a practice session with the new response keys. Although this is possible, further analysis suggests otherwise. Examining RTs from just Epoch 9, we see that there was no evidence of $\mathrm{CC}$ for either target-present or -absent trials, $t(23)=1.1$, n.s., and $t(23)=0.3$, n.s., respectively. Here, participants would have become accustomed to the new response keys, so if it was just a matter of practice, a CC effect should have reemerged. Instead, it seems that changing the overall response type from a discrimination task (i.e., is the $\mathrm{T}$ facing left or right?) to a detection task (i.e., is the $\mathrm{T}$ present or absent?) removed the CC effect. We discuss this further in the General Discussion.

\section{General discussion}

Contextual cuing has been found using a number of different display types. When certain aspects of a display are repeated, responses to targets are facilitated, as compared to when the display is not repeated. This can occur with repetition of the target-distractor configuration (Chun \& Jiang, 1998), global background color (Kunar et al., 2006), photograph of a naturalistic scene (Brockmole, Castelhano, \& Henderson, 2006; Brockmole \& Henderson, 2006; Ehinger \& Brockmole, 2008), or covariation with other objects (Chun \& Jiang, 1999). The present article investigated what happens to $\mathrm{CC}$ when there is no target present in configural $\mathrm{CC}$ displays. The results show that no $\mathrm{CC}$ effect is observed on target-absent displays, and the inclusion of absent trials, within a given context, also disrupts $\mathrm{CC}$ on target-present trials.
Previous results have shown that when a target is present, CC speeds RTs (e.g., Chun \& Jiang, 1998). Brady and Chun (2007; see also Jiang \& Wagner, 2004; Olson \& Chun, 2002) suggested that the relationship between the target and its immediately surrounding distractor items is what is important. Consistent with this idea, our data showed that a CC effect did not emerge in configural CC when the target was absent. Furthermore, these target-distractor associations appeared to be vulnerable whenever a distractor appeared in a likely target position.

In a related study, Jungé, Scholl, and Chun (2007) found that increasing the amount of noise in a CC study led to a disruption of contextual learning. In a condition in which participants were first shown a set of unrepeated trials that did not cue the target ("noise trials"), followed by repeated trials that did cue the target location ("signal trials"), no CC was observed. Jungé et al. suggested that if the visual system failed to pick up any display regularities initially (with this increased noise), it might conclude that no predictive information was in the display. A similar interpretation could be applied to our data. With the introduction of absent trials, and even more so when the same configuration could be used on present and absent trials, the system might conclude that it is "cheaper" to simply search the display de novo than to retrieve the learned context (Oliva et al., 2004; Wolfe et al., 2000; see also Kunar et al., 2008a, who showed that search through memory was less efficient than search from vision).

Interestingly, when Jungé et al. (2007) presented the signal displays first, before the noise displays, a $\mathrm{CC}$ effect emerged. Our results show that even well-learned CC can be disrupted by the subsequent introduction of absent trials, at least when distractors can appear in previously reliable target locations. The different fates of previously learned $\mathrm{CC}$ in these two studies might be due to differences in methodology. In Jungé et al.'s study, the "noise" trials did not share the same configuration as the "signal" trials, whereas in our study, the configurations that had signaled the location of the target on $100 \%$ of trials became imperfect predictors after the introduction of the absent trials. Perhaps any RT benefit of previously having learned the location of the target was negated by the extra cost of searching elsewhere when the target was not present and was replaced by a distractor. In these instances, the visual system might choose a strategy that favors searching the display rather than retrieving the learned configural cues (see also Kunar et al., 2008a; Makovski \& Jiang, 2010; Oliva et al., 2004; Wolfe et al., 2000).

The results from Experiment 5 also give us an insight into whether a guidance or response selection mechanism might be responsible for CC. In this experiment, contextual knowledge of the target's location had been previously 
learned. However, when the response changed from a target discrimination task (is the $\mathrm{T}$ facing left or right?) to a target detection task (is the T present or absent?), the CC effect was no longer there. An attentional guidance account of CC would not predict this result. Instead, the repeated display would guide attention to the target location, and so, at the very least, a facilitation effect should still occur in targetpresent trials. It did not.

On the other hand, the data can be explained by a responseselection account. Participants had previously learned to respond to the orientation of a $\mathrm{T}$, but this facilitation would not necessarily translate to a different and novel response dimension (see, e.g., Cohen \& Shoup, 1997; Feintuch \& Cohen, 2002, who suggested that different perceptual dimensions have different response-selection processes). In this case, a repeated display that had previously benefited orientation responses would not facilitate "present" or "absent" responses. This is what we observed.

\section{Conclusion}

In sum, contextual information can often facilitate response to a target. However, there are limits to when we encode and use that contextual information. This article has shown that configural contextual knowledge is only learned when it leads to the target on target-present trials. When the target is not there, local context does not seem to be treated as useful information.

Author Note This research was supported by a grant from the National Institutes of Health to J.M.W. (MH56020). We thank Kristin Michod, Stephen Flusberg, and Craig Scott for their assistance with data collection. We also thank Yuhong Jiang and James Brockmole for comments on an earlier version of the manuscript.

\section{References}

Biederman, I. (1972). Perceiving real-world scenes. Science, 177, 7780. doi:10.1126/science.177.4043.77

Brady, T. F., \& Chun, M. M. (2007). Spatial constraints on learning in visual search: Modeling contextual cuing. Journal of Experimental Psychology. Human Perception and Performance, 33, 798-815.

Brainard, D. H. (1997). The psychophysics toolbox. Spatial Vision, 10, 443-446. doi:10.1163/156856897X00357

Brockmole, J. R., Castelhano, M. S., \& Henderson, J. M. (2006). Contextual cuing in naturalistic scenes: Global and local contexts. Journal of Experimental Psychology. Learning, Memory, and Cognition, 32, 699-706.

Brockmole, J. R., \& Henderson, J. M. (2006). Using real-world scenes as contextual cues during search. Visual Cognition, 13, 99-108.

Chun, M. M. (2000). Contextual cueing of visual attention. Trends in Cognitive Sciences, 4, 170-178.

Chun, M. M., \& Jiang, Y. (1998). Contextual cueing: Implicit learning and memory of visual context guides spatial attention. Cognitive Psychology, 36, 28-71. doi:10.1006/cogp. 1998.0681
Chun, M. M., \& Jiang, Y. (1999). Top-down attentional guidance based on implicit learning of visual covariation. Psychological Science, 10, 360-365. doi:10.1111/1467-9280.00168

Chun, M. M., \& Jiang, Y. (2003). Implicit, long-term spatial contextual memory. Journal of Experimental Psychology. Learning, Memory, and Cognition, 29, 224-234. doi:10.1037/02787393.29 .2 .224

Cohen, A., \& Shoup, R. (1997). Perceptual dimensional constraints on response selection processes. Cognitive Psychology, 32, 128-181.

Duncan, J., \& Humphreys, G. W. (1989). Visual search and stimulus similarity. Psychological Review, 96, 433-458. doi:10.1037/0033295X.96.3.433

Egeth, H. E., Virzi, R. A., \& Garbart, H. (1984). Searching for conjunctively defined targets. Journal of Experimental Psychology. Human Perception and Performance, 10, 32-39.

Ehinger, K. A., \& Brockmole, J. R. (2008). The role of color in visual search in real-world scenes: Evidence from contextual cuing. Perception \& Psychophysics, 70, 1366-1378. doi:10.3758/ PP.70.7.1366

Endo, N., \& Takeda, Y. (2004). Selective learning of spatial configuration and object identity in visual search. Perception \& Psychophysics, 66, 293-302.

Feintuch, U., \& Cohen, A. (2002). Visual attention and coactivation of response decisions for features from different dimensions. Psychological Science, 13, 361-369.

Hoffmann, J., \& Sebald, A. (2005). Local contextual cuing in visual search. Experimental Psychology, 52, 31-38.

Jiang, Y., \& Chun, M. M. (2001). Selective attention modulates implicit learning. Quarterly Journal of Experimental Psychology, 54A, 1105-1124. doi:10.1080/02724980042000516

Jiang, Y., \& Leung, A. W. (2005). Implicit learning of ignored visual context. Psychonomic Bulletin \& Review, 12, 100-106.

Jiang, Y., Song, J.-H., \& Rigas, A. (2005). High-capacity spatial contextual memory. Psychonomic Bulletin \& Review, 12, 524-529.

Jiang, Y., \& Wagner, L. C. (2004). What is learned in spatial contextual cuing-Configuration or individual locations? Perception \& Psychophysics, 66, 454-463.

Johnson, J. S., Woodman, G. F., Braun, E., \& Luck, S. J. (2007). Implicit memory influences the allocation of attention in visual cortex. Psychonomic Bulletin \& Review, 14, 834-839.

Jungé, J. A., Scholl, B. J., \& Chun, M. M. (2007). How is spatial context learning integrated over time? A primacy effect in contextual cueing. Visual Cognition, 15, 1-11.

Kunar, M. A., Flusberg, S., Horowitz, T. S., \& Wolfe, J. M. (2007). Does contextual cuing guide the deployment of attention? Journal of Experimental Psychology. Human Perception and Performance, 33, 816-828. doi:10.1037/0096-1523.33.4.816

Kunar, M. A., Flusberg, S. J., \& Wolfe, J. M. (2006). Contextual cuing by global features. Perception \& Psychophysics, 68, 1204-1216.

Kunar, M. A., Flusberg, S. J., \& Wolfe, J. M. (2008a). The role of memory and restricted context in repeated visual search. Perception \& Psychophysics, 70, 314-328.

Kunar, M. A., Flusberg, S. J., \& Wolfe, J. M. (2008b). Time to guide: Evidence for delayed attentional guidance in contextual cueing. Visual Cognition, 16, 804-825. doi:10.1080/13506280701751224

Lleras, A., \& Von Mühlenen, A. (2004). Spatial context and top-down strategies in visual search. Spatial Vision, 17, 465-482. doi:10.1163/1568568041920113

Makovski, T., \& Jiang, Y. V. (2010). Contextual cost: When a visualsearch target is not where it should be. Quarterly Journal of Experimental Psychology, 63, 216-225.

Oliva, A., Wolfe, J. M., \& Arsenio, H. C. (2004). Panoramic search: The interaction of memory and vision in search through a familiar scene. Journal of Experimental Psychology. Human Perception and Performance, 30, 1132-1146. 
Olson, I. R., \& Chun, M. M. (2002). Perceptual constraints on implicit learning of spatial context. Visual Cognition, 9, 273-302.

Pelli, D. G. (1997). The VideoToolbox software for visual psychophysics: Transforming numbers into movies. Spatial Vision, 10, 437-442. doi:10.1163/156856897X00366

Reber, A. S. (1989). Implicit learning and tacit knowledge. Journal of Experimental Psychology. General, 118, 219-235.

Schankin, A., \& Schubö, A. (2009). Cognitive processes facilitated by contextual cueing: Evidence from event-related brain potentials. Psychophysiology, 46, 668-679. doi:10.1111/j.14698986.2009.00807.x

Smyth, A. C., \& Shanks, D. R. (2008). Awareness in contextual cuing with extended and concurrent explicit tests. Memory \& Cognition, 36, 403-415. doi:10.3758/MC.36.2.403

Theeuwes, J. (1992). Perceptual selectivity for color and form. Perception \& Psychophysics, 51, 599-606.
Treisman, A. M., \& Gelade, G. (1980). A feature-integration theory of attention. Cognitive Psychology, 12, 97-136. doi:10.1016/00100285(80)90005-5

Tseng, Y., \& Li, C. R. (2004). Oculomotor correlates of context-guided learning in visual search. Perception \& Psychophysics, 66, 13631378.

Wolfe, J. M., Cave, K. R., \& Franzel, S. L. (1989). Guided search: An alternative to the feature integration model for visual search. Journal of Experimental Psychology. Human Perception and Performance, 15, 419-433.

Wolfe, J. M., Klempen, N., \& Dahlen, K. (2000). Postattentive vision. Journal of Experimental Psychology. Human Perception and Performance, 26, 693-716. doi:10.1037/0096-1523.26.2.693

Yantis, S., \& Jonides, J. (1984). Abrupt visual onsets and selective attention: Evidence from visual search. Journal of Experimental Psychology. Human Perception and Performance, 10, 601-621. 